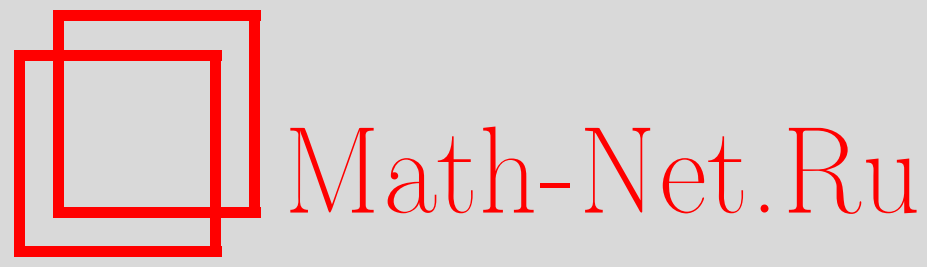

Д. Р. Яфаев, Коммутаторный метод диагонализации операторов Ганкеля, Функи. анализ и его прил., 2010, том 44, выпуск 4, 65-79

DOI: https://doi.org/10.4213/faa3019

Использование Общероссийского математического портала MathNet.Ru подразумевает, что вы прочитали и согласны с пользовательским соглашением

http://www.mathnet.ru/rus/agreement

Параметры загрузки:

IP: 54.196 .121 .252

26 апреля 2023 г., 16:11:16

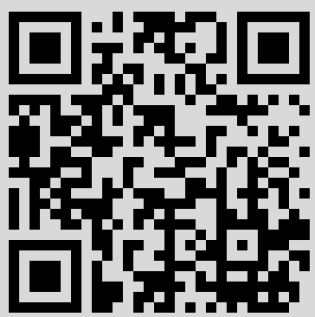




\title{
Коммутаторный метод диагонализации операторов Ганкеля
}

\author{
(c) 2010. Д. Р. ЯФАЕВ
}

Посвящается памяти М. Ш. Бирмана

Мы предлагаем метод явной диагонализации некоторых операторов Ганкеля. Этот метод позволяет дать новые доказательства классических результатов о диагонализации ганкелевых операторов с абсолютно непрерывным спектром. Он приводит также к новым результатам. Наш подход основан на коммутации оператора Ганкеля с некоторым дифференциальным оператором второго порядка.

\section{§1. Введение}

1.1. Операторы Ганкеля могут быть определены (см., например, книгу [8]) как интегральные операторы в пространстве $L^{2}\left(\mathbb{R}_{+}\right)$с ядрами, зависящими только от суммы переменных. Таким образом, ганкелев оператор $A$ задается формулой

$$
(A f)(x)=\int_{0}^{\infty} a(x+y) f(y) d y .
$$

Конечно, $A$ самосопряжен, если $a=\bar{a}$. Если

$$
\int_{0}^{\infty}|a(x)|^{2} x d x<\infty
$$

то $A$ принадлежит классу Гильберта-Шмидта. Это условие выполняется, если, например, функция $a$ непрерывна, не слишком сингулярна при $x=0$ и достаточно быстро убывает при $x \rightarrow \infty$. Напротив, если $a(x) \sim a_{0} x^{-1}$ при $x \rightarrow 0$ или (и) $a(x) \sim a_{\infty} x^{-1}$ при $x \rightarrow \infty$, то оператор $A$ перестает быть компактным, хотя и остается ограниченным. Общая философия (см. работу Хоуленда [11]) состоит в том, что каждая из этих сингулярностей порождает ветвь $\left[0, a_{0} \pi\right]$ или (и) $\left[0, a_{\infty} \pi\right]$ простого абсолютно непрерывного спектра.

Имеется очень мало примеров, когда оператор $A$ удается явно диагонализовать, т. е. найти его точные собственные функции. Первый такой результат принадлежит Мелеру [6], рассмотревшему случай $a(x)=(x+2)^{-1}$. Он показал, что функции

$$
\begin{aligned}
\psi_{k}(x) & =(k \operatorname{th} \pi k)^{1 / 2} P_{-1 / 2+i k}(x+1), & & k>0, \\
\lambda & =\pi / \operatorname{ch} \pi k, & & k>0,
\end{aligned}
$$

где $P_{-1 / 2+i k}-$ функция Лежандра (см. [2, гл. 3]), удовлетворяют уравнениям $A \psi_{k}=\lambda \psi_{k}$. Функции $\psi_{k}$ обычно параметризуются квазиимпульсом $k$, связан-

3 Функциональный анализ и его приложения, т. 44, вып. 4 
ным с $\lambda=\lambda(k)$ формулой (1.3). Оператор $U: L^{2}\left(\mathbb{R}_{+}\right) \rightarrow L^{2}\left(\mathbb{R}_{+}\right)$, определенный ${ }^{1)}$ равенством

$$
(U f)(k)=\int_{0}^{\infty} \psi_{k}(x) f(x) d x,
$$

унитарен. Так как $\lambda(k)$ - взаимно однозначное отображение полупрямой $\mathbb{R}_{+}$ на $(0, \pi)$ и $(U A f)(k)=\lambda(k) f(k)$, то спектр оператора $A$ простой, абсолютно непрерывный и совпадает с интервалом $[0, \pi]$.

Ниже мы используем термин «собственная функция» для функции $\psi_{k}$ (хотя $\left.\psi_{k} \notin L^{2}\left(\mathbb{R}_{+}\right)\right)$, такой, что $A \psi_{k}=\lambda \psi_{k}$ для спектрального параметра $\lambda$ из непрерывного спектра оператора $A$. По определению мы считаем, что собственные функции $\psi_{k}$ непрерывного спектра ортогональны, нормированы и множество всех $\psi_{k}$ полно, если соответствующий оператор (1.4) унитарен (при отсутствии у $A$ точечного спектра).

Следующий результат принадлежит В. Магнусу, рассмотревшему случай $a(x)=x^{-1} e^{-x / 2}$. Более общий результат того же типа был получен М. Розенблюмом, диагонализовавшим оператор $A$ с ядром

$$
a(x)=\Gamma(1+\beta) x^{-1} W_{-\beta, 1 / 2}(x), \quad \beta \in \mathbb{R}, \beta \neq-1,-2, \ldots,
$$

где $W_{-\beta, 1 / 2}$ - функция Уиттекера (см. [2, гл. 6]), а Г - гамма-функция. Отметим, что $W_{0,1 / 2}(x)=e^{-x / 2}$. Спектр оператора $A$ с таким ядром по-прежнему простой, и с точностью до конечного числа собственных значений он абсолютно непрерывен и совпадает с интервалом $[0, \pi]$. «Нормированные собственные функции» оператора $A$ выражаются через функции Уиттекера:

$$
\psi_{k}(x)=(2 \pi)^{-1} \sqrt{k|\Gamma(1 / 2-i k+\beta)| \operatorname{sh} 2 \pi k} x^{-1} W_{-\beta, i k}(x), \quad k>0 .
$$

Отметим, что функция $a(x)=(x+2)^{-1}$ сингулярна при $x=\infty$ и собственные функции (1.2) ведут себя как линейные комбинации функций $x^{-1 / 2 \pm i k}$ при $x \rightarrow \infty$, в то время как функция (1.5) сингулярна при $x=0$ и собственные функции (1.6) ведут себя как линейные комбинации тех же самых функций $x^{-1 / 2 \pm i k}$, но при $x \rightarrow 0$.

Наконец, упомянем простой случай $a(x)=x^{-1}$, когда оператор $A$ прямо диагонализуется (см. работу Карлемана [4]) преобразованием Меллина. В этом случае спектр оператора $A$ имеет кратность 2 (из-за сингулярностей функции $a(x)$ сразу при $x=0$ и при $x=\infty)$, абсолютно непрерывен и совпадает с интервалом $[0, \pi]$. Собственные функции оператора Карлемана равняются $x^{-1 / 2 \pm i k}$ (с точностью до нормировки).

Подчеркнем аналогию теорий сингулярных дифференциальных операторов и ганкелевых операторов с сингулярными ядрами. Таким образом, функции $x^{-1 / 2 \pm i k}$ играют (как при $x \rightarrow \infty$, так и при $x \rightarrow 0$ ) для ганкелевых операторов роль экспоненциальных функций $e^{ \pm i k x}$ для дифференциальных операторов второго порядка. С этой точки зрения оператор Карлемана играет роль оператора $-d^{2} / d x^{2}$ в пространстве $L^{2}(\mathbb{R})$.

1) Точное определение оператора $U$ может быть дано через соответствующую полуторалинейную форму. 
1.2. По мнению автора естественное объяснение того, почему в описанных выше случаях собственные функции оператора Ганкеля удается найти явно, отсутствовало. Наш подход показывает, что все диагонализуемые ганкелевы операторы $A$ коммутируют с дифференциальными операторами

$$
L=-\frac{d}{d x}\left(x^{2}+\gamma x\right) \frac{d}{d x}+\alpha x^{2}+\beta x
$$

при подходящих значениях параметров $\alpha \geqslant 0, \beta \in \mathbb{R}$ и $\gamma \geqslant 0$. Таким образом, операторы $A$ и $L$ имеют общие собственные функции, что и позволяет диагонализовать оператор $A$.

Можно надеяться, что коммутаторный метод окажется применимым и к другим ядрам $a$. В настоящей работе в разд. 4.4 мы применяем коммутаторный метод для нахождения собственных функций в новом случае - для оператора Ганкеля с ядром

$$
a(x)=\sqrt{\frac{8}{x}} K_{1}(\sqrt{8 x})
$$

где $K_{1}$ - функция Макдональда (см. [2, гл. 7]). Аналогично функции (1.5) эта функция убывает экспоненциально при $x \rightarrow \infty$ и $a(x) \sim x^{-1}$ при $x \rightarrow 0$. В качестве примера другого типа отметим ганкелевы операторы с регулярными ядрами; такие операторы компактны.

Заметим, что оператор (1.7) при $\gamma=0$ и $\alpha>0$ уже рассматривался в [9]. В этой работе Розенблюм исходил из тождества

$$
\Gamma(1+\beta) \int_{0}^{\infty}(x+y)^{-1} W_{-\beta, 1 / 2}(x+y) y^{-1} W_{-\beta, i k}(y) d y=\frac{\pi}{\operatorname{ch} \pi k} x^{-1} W_{-\beta, i k}(x),
$$

найденного ранее Шанкером в [12]. Это тождество показывает, что функции (1.6) являются собственными функциями ганкелева оператора с ядром (1.5). Розенблюм заметил, что функции (1.6) являются также собственными функциями оператора (1.7) для $\gamma=0$ и $\alpha=1 / 4$. Поскольку собственные функции самосопряженного дифференциального оператора $L$ ортогональны и полны, то же самое верно относительно собственных функций ганкелева оператора $A$ с ядром (1.5). Это приводит к диагонализации этого оператора.

Наш подход состоит несколько в другом. Мы устанавливаем соотношение $L A=A L$, из которого следует, что собственные функции операторов $L$ и $A$ совпадают. В частности, тождество (1.9) возникает вне рамок теории специальных функций.

Хорошо известно, что интегрируемость дифференциальных уравнений второго порядка в терминах специальных функций имеет глубокую теоретикогрупповую интерпретацию (см., например, книгу Виленкина [3]). Что касается операторов Ганкеля, то диагонализация оператора Карлемана является, конечно, следствием его инвариантности относительно группы растяжений. Соотношение $L A=A L$ означает, что оператор $A$ инвариантен относительно группы $\exp (-i t L)$. Однако, в отличие от оператора Карлемана, для других операторов Ганкеля такая инвариантность не выглядит очевидной.

Коммутаторная схема излагается в 2 , а конкретные примеры ядер, сингулярных при $x=\infty$ и $x=0$, обсуждаются соответственно в $\S 3$ и $\S 4$. Операторы Ганкеля с регулярными ядрами рассматриваются в $\S 5$. 


\section{§2. Коммутаторный метод}

2.1. Сначала мы рассматриваем оператор $L$, определенный формулой (1.7), как дифференциальный оператор на классе $C^{2}\left(\mathbb{R}_{+}\right)$, но позднее он будет определен как самосопряженный оператор в пространстве $L^{2}\left(\mathbb{R}_{+}\right)$. Пусть оператор $A$ задается формулой (1.1), где $a \in C^{2}\left(\mathbb{R}_{+}\right)$.

Прокоммутируем операторы $A$ и $L$. Пусть $f \in C^{2}\left(\mathbb{R}_{+}\right)$, и предположим, что

$$
\lim _{y \rightarrow 0}\left(y^{2}+\gamma y\right) f(y)=\lim _{y \rightarrow 0}\left(y^{2}+\gamma y\right) f^{\prime}(y)=0
$$

и

$$
\lim _{y \rightarrow \infty} a^{\prime}(x+y)\left(y^{2}+\gamma y\right) f(y)=\lim _{y \rightarrow \infty} a(x+y)\left(y^{2}+\gamma y\right) f^{\prime}(y)=0
$$

для всех $x \geqslant 0$. Тогда, интегрируя по частям, найдем, что

$$
(A L f)(x)=\int_{0}^{\infty}\left(-\frac{\partial}{\partial y}\left(\left(y^{2}+\gamma y\right) a^{\prime}(x+y)\right)+a(x+y)\left(\alpha y^{2}+\beta y\right)\right) f(y) d y .
$$

Отсюда следует, что

$$
((L A-A L) f)(x)=\int_{0}^{\infty} q(x, y) f(y) d y,
$$

где

$$
\begin{aligned}
q(x, y)=- & \frac{\partial}{\partial x}\left(\left(x^{2}+\gamma x\right) a^{\prime}(x+y)\right)+\frac{\partial}{\partial y}\left(\left(y^{2}+\gamma y\right) a^{\prime}(x+y)\right) \\
& \quad+\left(\alpha x^{2}-\alpha y^{2}+\beta x-\beta y\right) a(x+y) \\
= & (x-y)\left(-(z+\gamma) a^{\prime \prime}(z)-2 a^{\prime}(z)+(\alpha z+\beta) a(z)\right)
\end{aligned}
$$

и $z=x+y$. Таким образом, мы приходим к следующему результату общего характера.

Теорема 2.1. Предположим, что ядро а оператора Ганкеля А удовлетворяет дифференциальному уравнению

$$
-(x+\gamma) a^{\prime \prime}(x)-2 a^{\prime}(x)+(\alpha x+\beta) a(x)=0 .
$$

Пусть $f \in C^{2}\left(\mathbb{R}_{+}\right)$, и пусть выполняются условия (2.1) и (2.2). Тогда

$$
(L A-A L) f=0 .
$$

Отметим, что после замены переменных

$$
a(x)=(x+\gamma)^{-1} b(x+\gamma)
$$

в (2.3) мы получаем уравнение Шрёдингера с кулоновским потенциалом

$$
-b^{\prime \prime}(r)+(\beta-\alpha \gamma) r^{-1} b(r)=-\alpha b(r) .
$$

2.2. В конкретных примерах мы будем использовать теорему 2.1 следующим образом. Если $L$ самосопряжен и имеет простой спектр, то равенство $L A=A L$ показывает, что $A-$ функция $\mathrm{F}$ от $L$, т.е. операторы $A$ и $L$ имеют общие собственные функции. Для вычисления функции $\mathrm{F}$ мы поступаем следующим образом. Предположим, что вещественная функция $\psi_{\mu}$ удовлетворяет условиям (2.1), (2.2) и уравнению

$$
-\left(\left(x^{2}+\gamma x\right) \psi_{\mu}^{\prime}(x)\right)^{\prime}+\left(\alpha x^{2}+\beta x\right) \psi_{\mu}(x)=\mu \psi_{\mu}(x) .
$$


Тогда, согласно равенству (2.4), тому же самому уравнению удовлетворяет функция $A \psi_{\mu}$ и, следовательно, при некоторых числах $\lambda=\lambda_{\mu}$ и $\check{\lambda}=\check{\lambda}_{\mu}$

$$
\left(A \psi_{\mu}\right)(x)=\lambda \psi_{\mu}(x)+\check{\lambda} \check{\psi}_{\mu}(x),
$$

где $\check{\psi}_{\mu}$ - решение уравнения $L \check{\psi}_{\mu}=\mu \check{\psi}_{\mu}$, линейно независимое с $\psi_{\mu}$. Далее, сравнивая асимптотики функций $\psi_{\mu}(x), \check{\psi}_{\mu}(x)$ и $\left(A \psi_{\mu}\right)(x)$ при $x \rightarrow 0$ и при $x \rightarrow \infty$, мы видим, что $\check{\lambda}=0$, и находим $\lambda=\mathrm{F}(\mu)$ как функцию от $\mu$. Наконец, если $\psi_{\mu}$ принадлежит области определения какой-либо самосопряженной реализации дифференциального оператора $L$, то при подходящей нормировке функций $\psi_{\mu}$ система всех функций $\psi_{\mu}$ ортогональна и полна. В этом случае $A=\mathrm{F}(L)$. Отметим, что такой подход позволяет избежать точного определения коммутаторов и ссылок на результаты из функционального анализа.

Оказывается, что во всех наших приложениях $\mathrm{F}(\mu)=\pi / \operatorname{ch}(\pi \sqrt{\mu-1 / 4})$ и, следовательно,

$$
A=\pi / \operatorname{ch}(\pi \sqrt{L-1 / 4}) .
$$

На самом деле несколько удобнее параметризовать собственные функции квазиимпульсом $k>0$, связанным с $\mu$ и $\lambda$ формулами

$$
\mu=k^{2}+1 / 4 \in(1 / 4, \infty), \quad \lambda=\pi / \operatorname{ch} \pi k \in(0, \pi) .
$$

Подчеркнем, что $\psi_{k}(x), \psi_{\mu}(x)$ и $\psi_{\lambda}(x)$ обозначают одну и ту же функцию при условии, что параметры $k, \mu$ и $\lambda$ связаны формулами (2.9).

Оператор $U$, определенный соотношением (1.4), унитарен, и оператор $U A U^{*}$ действует в $L^{2}\left(\mathbb{R}_{+}\right)$как умножение на функцию $\lambda(k)=\pi / \operatorname{ch} \pi k$. Действительно, согласно теореме Фубини, из уравнения $A \psi_{k}=\lambda(k) \psi_{k}$ вытекает, что при $g \in$ $C_{0}^{\infty}\left(\mathbb{R}_{+}\right)$

$$
\begin{aligned}
\left(A U^{*} g\right)(x) & =\int_{0}^{\infty} d k g(k) \int_{0}^{\infty} d y a(x+y) \psi_{k}(y) \\
& =\int_{0}^{\infty} \lambda(k) \psi_{k}(x) g(k) d k=\left(U^{*}(\lambda g)\right)(x) .
\end{aligned}
$$

Это эквивалентно соотношению

$$
(U A f)(k)=\lambda(k)(U f)(k) \quad \forall f \in L^{2}\left(\mathbb{R}_{+}\right) .
$$

Поскольку $\lambda: \mathbb{R}_{+} \rightarrow(0, \pi)$ - взаимно однозначное отображение, оператор $A$ имеет простой абсолютно непрерывный спектр, совпадающий с отрезком $[0, \pi]$.

При реализации этой схемы случаи сингулярностей при $x=\infty$, когда $\gamma>0$, и при $x=0$, когда $\gamma=0$, удобно рассматривать порознь.

\section{§3. Сингулярность на бесконечности}

3.1. Положим $\gamma=2$. Сначала мы предполагаем, что $\alpha=\beta=0$. Тогда функция $a(x)=(x+2)^{-1}$ удовлетворяет уравнению $(2.3)$, и соответствующий оператор $L$ имеет вид

$$
L=-\frac{d}{d x} p(x) \frac{d}{d x}, \quad \text { где } p(x)=x^{2}+2 x .
$$


Обозначим через $P_{\nu}(z)$ и $Q_{\nu}(z)$ функции Лежандра первого и второго рода (см., например, [2, гл. 3]). Они определяются как решения уравнения

$$
\left(1-z^{2}\right) u^{\prime \prime}(z)-2 z u^{\prime}(z)+\nu(\nu+1) u(z)=0, \quad z>1,
$$

удовлетворяющие условиям $P_{\nu}(1)=1$ и $Q_{\nu}(z)=-2^{-1} \ln (z-1)+c_{\nu}$ при $z \rightarrow$ $1+0$ (значение константы $c_{\nu}$ несущественно). Тогда функции $P_{-1 / 2+i k}(x+1)$ и $Q_{-1 / 2+i k}(x+1)$ удовлетворяют уравнению $L u=\left(k^{2}+1 / 4\right) u$. Отметим также (см. формулы $(2.10 .2)$ и $(2.10 .5)$ из [2]), что

$$
P_{-1 / 2+i k}(x+1)=m(k) x^{-1 / 2+i k}+\overline{m(k)} x^{-1 / 2-i k}+O\left(x^{-3 / 2}\right), \quad x \rightarrow \infty,
$$

где

$$
m(k)=\frac{\Gamma(i k)}{\sqrt{2 \pi} \Gamma(1 / 2+i k)} 2^{i k} .
$$

Оператор $L$ симметричен в пространстве $L^{2}\left(\mathbb{R}_{+}\right)$на области определения $C_{0}^{\infty}\left(\mathbb{R}_{+}\right)$, но не является существенно самосопряженным. Поскольку обе функции $P_{-1 / 2+i k}(x+1)$ и $Q_{-1 / 2+i k}(x+1)$ принадлежат $L^{2}$ в окрестности точки $x=0$, дефектные числа оператора $L$ равны $(1,1)$. Одно из самосопряженных расширений оператора $L$ с $C_{0}^{\infty}\left(\mathbb{R}_{+}\right)$(оно также обозначается через $L$ ) определено на области $\mathscr{D}(L)$, состоящей из функций $f(x)$, принадлежащих классу Соболева $\mathrm{H}_{\mathrm{loc}}^{2}\left(\mathbb{R}_{+}\right)$и удовлетворяющих граничным условиям

$$
\exists \lim _{x \rightarrow 0} f(x), \quad f^{\prime}(x)=o\left(x^{-1 / 2}\right), \quad x \rightarrow 0
$$

(мы называем такие граничные условия регулярными); предполагается также, что $f \in L^{2}\left(\mathbb{R}_{+}\right)$и $L f \in L^{2}\left(\mathbb{R}_{+}\right)$. В самом деле, прямое интегрирование по частям показывает, что оператор $L$ симметричен. Далее, применяя подходящую функцию Грина, найдем, что для всех $h \in L^{2}\left(\mathbb{R}_{+}\right)$уравнение $\left(p f^{\prime}\right)^{\prime}=h$ имеет решение, удовлетворяющее условию (3.3). Таким образом, область значений оператора $L$ совпадает с $L^{2}\left(\mathbb{R}_{+}\right)$и, следовательно, $L$ самосопряжен (ср. $\S 132$, п. II, из [1]).

3.2. Для изучения оператора $L$ удобно сделать стандартную (см., например, книгу Титчмарша [10]) замену переменных. Положим

$$
t=\omega(x)=\int_{0}^{x} p(y)^{-1 / 2} d y \quad \text { и } \quad f(x)=\omega^{\prime}(x)^{1 / 2} \tilde{f}(\omega(x))=:(F \tilde{f})(x) .
$$

Оператор $F$ унитарен в пространстве $L^{2}\left(\mathbb{R}_{+}\right)$, а оператор $\widetilde{L}=F^{-1} L F$ действует по формуле $\widetilde{L}=-d^{2} / d t^{2}+q(\eta(t))$, где $q(x)=-16^{-1} p(x)^{-1} p^{\prime}(x)^{2}+4^{-1} p^{\prime \prime}(x)$ и $\eta=\omega^{-1}-$ функция, обратная к $\omega$ (так что $\left.x=\eta(t)\right)$.

Если $p(x)=x^{2}+2 x$, то

$$
\omega(x)=2 \ln \left(x^{1 / 2}+(x+2)^{1 / 2}\right)-\ln 2
$$

и, следовательно,

$$
\widetilde{L}=-\frac{d^{2}}{d t^{2}}+\tilde{q}(t)+\frac{1}{4}
$$

где $\tilde{q}(t)=-4^{-1}\left(\eta^{2}(t)+2 \eta(t)\right)^{-1}$. Так как $\omega(x)=(2 x)^{1 / 2}+O(x)$ при $x \rightarrow 0$ и $\omega(x)=\ln (2 x)+O\left(x^{-1}\right)$ при $x \rightarrow \infty$, то $\eta(t) \sim t^{2} / 2$ при $t \rightarrow 0$ и $\eta(t) \sim e^{t} / 2$ при $t \rightarrow \infty$. Отсюда следует, что $\tilde{q}(t) \sim-\left(4 t^{2}\right)^{-1}$ при $t \rightarrow 0$ и $\tilde{q}(t)=O\left(e^{-t}\right)$ 
при $t \rightarrow \infty$. Отметим, что оператор $\widetilde{L}$ самосопряжен на области определения $\mathscr{D}(\widetilde{L})$, состоящей из функций $\tilde{f}(t)$, принадлежащих классу Соболева $\mathrm{H}_{\text {loc }}^{2}\left(\mathbb{R}_{+}\right)$, удовлетворяющих граничным условиям

$$
\exists \lim _{t \rightarrow 0} t^{-1 / 2} \tilde{f}(t), \quad \tilde{f}^{\prime}(t)-(2 t)^{-1} \tilde{f}(t)=o\left(t^{1 / 2}\right), \quad t \rightarrow 0,
$$

и таких, что $\tilde{f} \in L^{2}\left(\mathbb{R}_{+}\right), \tilde{L} \tilde{f} \in L^{2}\left(\mathbb{R}_{+}\right)$.

Все обычные результаты спектральной теории и теории рассеяния применяются сначала к оператору $\widetilde{L}$, а затем используются для оператора $L$. Оператор $\widetilde{L}$ имеет простой абсолютно непрерывный спектр, совпадающий с интервалом $[1 / 4, \infty)$. Он не имеет собственных значений, так как уравнение $\widetilde{L} \tilde{u}=\mu \tilde{u}$ или, что эквивалентно, $L u=\mu u$ при $\mu \in \mathbb{R}$ не имеет решений из $L^{2}\left(\mathbb{R}_{+}\right)$, удовлетворяющих регулярным граничным условиям в нуле. Оператор $\widetilde{L}$ может быть диагонализован (см., например, [10], [13]) следующим образом. Пусть $\tilde{u}_{k}(t), k>0$, - вещественное решение уравнения

$$
\widetilde{L} \tilde{u}_{k}=\left(k^{2}+1 / 4\right) \tilde{u}_{k},
$$

удовлетворяющее граничным условиям (3.7). Оно имеет асимптотику

$$
\tilde{u}_{k}(t)=m(k) e^{i k t}+\overline{m(k)} e^{-i k t}+o(1)
$$

при $t \rightarrow \infty$. Тогда оператор $\widetilde{U}$, определенный соотношением

$$
(\widetilde{U} \tilde{f})(k)=(2 \pi)^{-1 / 2}|m(k)|^{-1} \int_{0}^{\infty} \tilde{u}_{k}(t) \tilde{f}(t) d t
$$

унитарен в пространстве $L^{2}\left(\mathbb{R}_{+}\right)$и $(\widetilde{U} \widetilde{L} \tilde{f})(k)=\left(k^{2}+1 / 4\right)(\widetilde{U} \tilde{f})(k)$.

Сделаем замену переменных (3.4) и положим $U=F \widetilde{U} F^{-1}$. Отметим, что

$$
(2 \pi)^{-1 / 2}|m(k)|^{-1}=\sqrt{k \operatorname{th} \pi k},
$$

где функция $m(k)$ определяется равенством (3.2). Отсюда следует, что оператор $U$, заданный соотношением

$$
(U f)(k)=\sqrt{k \operatorname{th} \pi k} \int_{0}^{\infty} P_{-1 / 2+i k}(x+1) f(x) d x,
$$

унитарен в пространстве $L^{2}\left(\mathbb{R}_{+}\right)$и

$$
(U L f)(k)=\left(k^{2}+1 / 4\right)(U f)(k) .
$$

3.3. Вернемся к ганкелеву оператору $A$. Заметим, что функция $P_{-1 / 2+i k}(x+1)$ удовлетворяет ${ }^{1)}$ обоим граничным условиям (2.1) и (2.2). Из теоремы 2.1 вытекает, что

$$
\int_{0}^{\infty}(x+y+2)^{-1} P_{-1 / 2+i k}(y+1) d y=\lambda P_{-1 / 2+i k}(x+1)+\check{\lambda} Q_{-1 / 2+i k}(x+1) .
$$

1) Мы должны выбирать регулярные граничные условия в нуле, так как функция $Q_{-1 / 2+i k}(x+1)$ не удовлетворяет второму граничному условию $(2.1)$. 
Рассматривая здесь предел $x \rightarrow 0$, мы видим, что $\check{\lambda}=0$. Затем мы находим предел при $x \rightarrow \infty$. Из (3.1) легко следует, что левая часть формулы (3.13) равна

$$
\begin{aligned}
2 \operatorname{Re}\left(m(k) \int_{0}^{\infty}(x+\right. & \left.y+2)^{-1} y^{-1 / 2+i k} d y\right)+O\left(x^{-1}\right) \\
& =2 \operatorname{Re}\left(m(k) x^{-1 / 2+i k} \int_{0}^{\infty}(t+1)^{-1} t^{-1 / 2+i k} d t\right)+O\left(x^{-1}\right),
\end{aligned}
$$

где мы положили $y=x t$. Сравнивая эту асимптотику с асимптотикой $(3.1)$ правой части формулы (3.13), получаем, что

$$
\lambda=\int_{0}^{\infty}(t+1)^{-1} t^{-1 / 2+i k} d t=\pi(\operatorname{ch} \pi k)^{-1}
$$

и, следовательно,

$$
\int_{0}^{\infty}(x+y+2)^{-1} P_{-1 / 2+i k}(y+1) d y=\pi(\operatorname{ch} \pi k)^{-1} P_{-1 / 2+i k}(x+1) .
$$

Это дает уравнение (2.10) с оператором $U$, определенным формулой (3.11). Поскольку оператор $U$ унитарен, мы пришли к результату Мелера [6].

Предложение 3.1. Оператор Ганкеля с ядром $a(x)=(x+2)^{-1}$ имеет простой абсолютно непрерывный спектр, совпадающий с интервалом $[0, \pi]$. Его нормированная собственная функиия, отвечающая спектральному параметру $\lambda=\pi(\operatorname{ch} \pi k)^{-1}$, определяется формулой (1.2).

Подчеркнем, что уравнение (3.15) было получено как прямое следствие коммутаторного метода, без использования результатов теории специальных функций.

\section{§4. Сингулярность в нуле}

В первых трех разделах мы рассматриваем оператор Ганкеля с ядром (1.5), а в разд. $4-$ с ядром (1.8). В обоих случаях $a(x) \sim x^{-1}$ при $x \rightarrow 0$ и $a(x)$ экспоненциально убывает при $x \rightarrow \infty$. Соответствующий оператор $L$ определяется формулой (1.7), где $\gamma=0$.

4.1. Отметим, что в случае $\gamma=0$ после замены переменных $\psi(x)=x^{-1} \varphi(x)$ в (2.7) мы вновь (ср. с уравнением (2.6)) получаем уравнение Шрёдингера

$$
-\varphi^{\prime \prime}(x)+\left(\alpha+\beta x^{-1}-\mu x^{-2}\right) \varphi(x)=0
$$

с кулоновским потенциалом, но с ненулевым орбитальным членом. Ниже мы полагаем $\alpha=1 / 4$.

Напомним, что функция Уиттекера $W_{-\beta, p}(x)$ может быть определена как решение уравнения (4.1), где $\mu=1 / 4-p^{2}$, такое, что

$$
W_{-\beta, p}(x)=x^{-\beta} e^{-x / 2}\left(1+O\left(x^{-1}\right)\right)
$$

при $x \rightarrow \infty$. Конечно, $W_{-\beta,-p}(x)=W_{-\beta, p}(x)$. В частности, функция $b(x)=$ $W_{-\beta, 1 / 2}(x)$ удовлетворяет уравнению $(2.6)$, где $\alpha=1 / 4$ и $\gamma=0$.

Что касается асимптотики при $x \rightarrow 0$ (см. §6.8 книги [2]), то

$$
W_{-\beta, i k}(x)=m(k) x^{1 / 2+i k}+\overline{m(k)} x^{1 / 2-i k}+O\left(x^{3 / 2}\right), \quad k>0, x \rightarrow 0,
$$


где

$$
m(k)=\Gamma(-2 i k)(\Gamma(1 / 2-i k+\beta))^{-1} .
$$

Если $p \geqslant 0$ и $-1 / 2+p+\beta \neq-1,-2, \ldots$, то при $x \rightarrow 0$

$$
\begin{aligned}
& W_{-\beta, p}(x) \sim \Gamma(2 p)(\Gamma(1 / 2+p+\beta))^{-1} x^{1 / 2-p}, \quad p>0, \\
& W_{-\beta, 0}(x) \sim-\Gamma(1 / 2+\beta) x^{1 / 2} \ln x .
\end{aligned}
$$

Если $-1 / 2+p+\beta=-n$, где $n=1,2, \ldots$, то, используя формулы (6.9.4) и (6.9.36) из [2], мы можем выразить функции Уиттекера в терминах полиномов Лагерра:

$$
W_{-\beta, p}(x)=(-1)^{n-1}(n-1) ! e^{-x / 2} x^{p+1 / 2} L_{n-1}^{2 p}(x) .
$$

Если $\gamma=0$ и $\alpha=1 / 4$, то

$$
L=-\frac{d}{d x} x^{2} \frac{d}{d x}+\frac{x^{2}}{4}+\beta x .
$$

Подчеркнем, что коэффициент $\beta$ может быть произвольным. Выясняется, что сильное вырождение функции $x^{2}$ при $x=0$ порождает ветвь абсолютно непрерывного спектра оператора $L$.

Сначала мы определим $L$ как самосопряженный оператор в пространстве $L^{2}\left(\mathbb{R}_{+}\right)$. Мы проверим, что он существенно самосопряжен на области определения $C_{0}^{\infty}\left(\mathbb{R}_{+}\right)$. Пусть ${ }^{1)}(F \tilde{f})(x)=x^{-1 / 2} \tilde{f}(\ln x)$. Тогда оператор $F: L^{2}(\mathbb{R}) \rightarrow$ $L^{2}\left(\mathbb{R}_{+}\right)$унитарен и оператор $\widetilde{L}=F^{-1} L F$ действует по формуле (3.6), где $\tilde{q}(t)=e^{2 t} / 4+\beta e^{t}$. Это дает нам стандартный оператор Штурма-Лиувилля в пространстве $\widetilde{\mathscr{H}}=L^{2}(\mathbb{R})$. Потенциал $\tilde{q}(t)$ стремится к 0 при $t \rightarrow-\infty$ и $\mathrm{K}+\infty$ при $t \rightarrow+\infty$. В частности, $\widetilde{L}$ существенно самосопряжен на $C_{0}^{\infty}(\mathbb{R})$, откуда следует существенная самосопряженность оператора $L$ на $C_{0}^{\infty}\left(\mathbb{R}_{+}\right)$в пространстве $L^{2}\left(\mathbb{R}_{+}\right)$. Таким образом, граничное условие в точке $x=0$ не нужно. Так как $\tilde{q}(t) \rightarrow+\infty$ при $t \rightarrow+\infty$, то квантовая частица может уходить только на $-\infty$. Поэтому спектр оператора $\widetilde{L}$ простой.

4.2. Разложение по собственным функциям оператора $\widetilde{L}$ может быть построено с помощью следующей стандартной процедуры (см., например, [13, разд. 5.4]). Как мы увидим ниже, помимо простого абсолютно непрерывного спектра, совпадающего с $[1 / 4, \infty)$, при $\beta<-1 / 2$ оператор $\widetilde{L}$ имеет конечное число простых собственных значений $\mu_{1}, \ldots, \mu_{N}, N=N(\beta)$, лежащих левее точки $1 / 4$. Обозначим через $\widetilde{\mathscr{H}}^{(p)}$ подпространство, натянутое на соответствующие собственные функции. Пусть $\tilde{u}_{k}(t), k>0$, - вещественное решение уравнения (3.8), принадлежащее $L^{2}\left(\mathbb{R}_{+}\right)$. Оно имеет асимптотику (3.9) при $t \rightarrow-\infty$ с функцией $m(k)$, которая вычисляется ниже. Тогда оператор $\widetilde{U}: \widetilde{\mathscr{H}} \rightarrow L^{2}\left(\mathbb{R}_{+}\right)$, определяемый уравнением (ср. с (3.10))

$$
(\tilde{U} \tilde{f})(k)=(2 \pi)^{-1 / 2}|m(k)|^{-1} \int_{-\infty}^{\infty} \tilde{u}_{k}(t) \tilde{f}(t) d t
$$

1) Отметим, что интеграл в (3.4) расходится для $p(x)=x^{2}$, и, следовательно, определение оператора $F$ нуждается в модификации. 
ограничен, $\left.\widetilde{U}\right|_{\widetilde{\mathscr{H}}(p)}=0$, отображение $\widetilde{U}: \widetilde{\mathscr{H}} \ominus \widetilde{\mathscr{H}^{(p)}} \rightarrow L^{2}\left(\mathbb{R}_{+}\right)$унитарно и выполняется уравнение

$$
(\widetilde{U} \widetilde{L} \tilde{f})(k)=\left(k^{2}+1 / 4\right)(\widetilde{U} \tilde{f})(k) .
$$

Функции $u_{k}(x)=x^{-1 / 2} \tilde{u}_{k}(\ln x), k>0$, удовлетворяют уравнению

$$
-\left(x^{2} u_{k}^{\prime}(x)\right)^{\prime}+4^{-1} x^{2} u_{k}(x)+\beta x u_{k}(x)=\left(k^{2}+1 / 4\right) u_{k}(x)
$$

и выражаются через функции Уиттекера:

$$
u_{k}(x)=x^{-1} W_{-\beta, i k}(x) .
$$

Из (4.3) следует, что функция $\tilde{u}_{k}(t)=e^{t / 2} u_{k}\left(e^{t}\right)$ имеет при $t \rightarrow-\infty$ асимптотику (3.9) с функцией $m(k)$, определяемой равенством (4.4). Вычисляя $|m(k)|$ и проводя в (4.4) замену переменных $t=\ln x$, получаем, что оператор $U=F \widetilde{U} F^{-1}$ удовлетворяет соотношению

$$
(U f)(k)=\pi^{-1} \sqrt{k \operatorname{sh} 2 \pi k}|\Gamma(1 / 2-i k+\beta)| \int_{0}^{\infty} x^{-1} W_{-\beta, i k}(x) f(x) d x .
$$

Он ограничен, $\left.U\right|_{\mathscr{H}(p)}=0$, отображение $U: \mathscr{H} \ominus \mathscr{H}^{(p)} \rightarrow L^{2}\left(\mathbb{R}_{+}\right)$унитарно, и справедливо уравнение (3.12). Здесь $\mathscr{H}^{(p)}$ - подпространство, натянутое на собственные функции $\psi_{1}, \ldots, \psi_{N}$ оператора $L$.

Вычислим эти функции. Функция $u_{p}(x)=x^{-1} W_{-\beta, p}(x)$ при $p \geqslant 0$ удовлетворяет уравнению (4.9), где роль $k^{2}$ играет $-p^{2}$. Ввиду (4.2) она принадлежит $L^{2}$ на бесконечности. Однако из асимптотик (4.5) следует, что она может принадлежать $L^{2}$ в окрестности точки $x=0$ лишь при $-1 / 2+p+\beta=-n$, где $n=1,2, \ldots$ Это возможно только при $\beta \geqslant-1 / 2$. Кроме того, при $\beta=-1 / 2$ обязательно $p=0$, однако в силу (4.6) функция $u_{0}$ не принадлежит $L^{2}$ (ни при каких $\beta$ ). Таким образом, если $\beta \geqslant-1 / 2$, то оператор $L$ имеет только абсолютно непрерывный спектр. Если $\beta<-1 / 2$, то он имеет также собственные значения $\mu_{n}=1 / 4-(|\beta|+1 / 2-n)^{2}$, где $n=1,2, \ldots$ и $n<|\beta|+1 / 2$ (с тем чтобы $p>0)$. Согласно формуле $(4.6)$, соответствующими собственными функциями являются

$$
\psi_{n}(x)=e^{-x / 2} x^{p-1 / 2} L_{n-1}^{2 p}(x), \quad p=|\beta|+1 / 2-n .
$$

4.3. Вернемся к оператору Ганкеля $A$ с ядром (1.5). Из (4.2) следует, что $a(x)$ экспоненциально убывает при $x \rightarrow \infty$, а из первой формулы (4.5), где $p=1 / 2$, следует, что $a(x) \sim x^{-1}$ при $x \rightarrow 0$. Заметим, что ввиду асимптотик (4.2) и (4.3) функция (4.10) удовлетворяет обоим граничным условиям (2.1) и (2.2). Таким образом, из теоремы 2.1 вытекает, что

$$
\int_{0}^{\infty} a(x+y) y^{-1} W_{-\beta, i k}(y) d y=\lambda(k) x^{-1} W_{-\beta, i k}(x)+\check{\lambda}(k) x^{-1} M_{-\beta, i k}(x),
$$

где функция Уиттекера $M_{-\beta, i k}$ - решение уравнения (2.6), экспоненциально растущее при $x \rightarrow \infty$. Поэтому, рассматривая в (4.12) предел $x \rightarrow \infty$, мы видим, что обязательно $\check{\lambda}(k)=0$. Затем мы берем предел при $x \rightarrow 0$ и используем 
асимптотику (4.3). Так как $a(x) \sim x^{-1}$ при $x \rightarrow 0$, то

$$
\begin{aligned}
\int_{0}^{\infty} a(x+y) y^{-1} W_{-\beta, i k}(y) d y & =2 \operatorname{Re}\left(m(k) \int_{0}^{\infty}(x+y)^{-1} y^{-1 / 2+i k} d y\right)+O(1) \\
& =2 \lambda(k) \operatorname{Re}\left(m(k) x^{-1 / 2+i k}\right)+O(1),
\end{aligned}
$$

где $\lambda(k)$ опять задается формулой (3.14). Это приводит к уравнению (1.9).

Остается вычислить собственные значения $\lambda_{1}, \ldots, \lambda_{N}$ оператора $A$. Соответствующие собственные функции определяются формулой (4.11). Будем исходить из уравнения (4.12), где роль $i k$ играет $p=|\beta|+1 / 2-n$. Как и раньше, рассматривая предел $x \rightarrow \infty$, получаем, что $\check{\lambda}_{n}=0$ и, следовательно,

$$
\int_{0}^{\infty} a(x+y) e^{-y / 2} y^{p-1 / 2} L_{n-1}^{2 p}(y) d y=\lambda_{n} e^{-x / 2} x^{p-1 / 2} L_{n-1}^{2 p}(x) .
$$

Из (1.5) и (4.2) вытекает, что левая часть здесь равна

$$
\Gamma(1+\beta) x^{-\beta-1} e^{-x / 2} \int_{0}^{\infty} e^{-y} y^{p-1 / 2} L_{n-1}^{2 p}(y) d y\left(1+O\left(x^{-1}\right)\right), \quad x \rightarrow \infty .
$$

Сопоставляя формулы (2.8.46) и (10.12.33) из [2], мы видим, что

$$
(n-1) ! \int_{0}^{\infty} e^{-y} y^{p-1 / 2} L_{n-1}^{2 p}(y) d y=\Gamma(p+n-1 / 2) .
$$

Вспомним также, что $L_{n-1}^{2 p}(x)$ - полином степени $n-1$ с коэффициентом $(-1)^{n-1} /(n-1)$ ! при $x^{n-1}$. Таким образом, из соотношения (4.13) следует, что

$$
\lambda_{n}=(-1)^{n} \pi / \sin \pi \beta, \quad n=1,2, \ldots, n<|\beta|+1 / 2 .
$$

Поскольку собственные функции оператора $L$ ортогональны и полны, мы приходим к результату Розенблюма [9].

Предложение 4.1. Оператор Ганкеля $A$ с ядром (1.5) имеет простой абсолютно непрерывный спектр, совпадающий с интервалом $[0, \pi]$. Его нормированная собственная функиия, отвечающая точке непрерывного спектра $\lambda=$ $\pi(\operatorname{ch} \pi k)^{-1}$, дается формулой

$$
\psi_{k}(x)=\pi^{-1} \sqrt{k \operatorname{sh} 2 \pi k}|\Gamma(1 / 2-i k+\beta)| x^{-1} W_{-\beta, i k}(x), \quad k>0 .
$$

Кроме того, если $\beta<-1 / 2$, то оператор $A$ имеет собственные значения (4.15) и соответствующие собственные функиии (4.11).

4.4. Теперь мы обратимся к оператору Ганкеля с сингулярным ядром (1.8), который, по-видимому, ранее в литературе не рассматривался. Напомним, что функция Макдональда определяется соотношением $K_{p}(z)=2^{-1} i e^{\pi i p / 2} H_{p}^{(1)}(i z)$, где $H_{p}^{(1)}$ - функция Ганкеля. Теперь функция $b(x)=x a(x)$ удовлетворяет уравнению Шрёдингера (2.3) при нулевой энергии $\alpha=0$ и константе связи $\beta=2$. Разумеется, мы могли бы взять любое $\beta>0$, но мы должны исключать отрицательные $\beta$, поскольку в этом случае функция $b(x)$ растет при $x \rightarrow \infty$.

Из хорошо известных свойств функции $H_{p}^{(1)}$ вытекает, что функция $(1.8)$ имеет асимптотику

$$
a(x)=4 \pi^{1 / 2} x^{-3 / 4} e^{-\sqrt{8 x}}\left(1+O\left(x^{-1 / 2}\right)\right)
$$


при $x \rightarrow \infty$ и $a(x) \sim x^{-1}$ при $x \rightarrow 0$.

Соответствующий оператор

$$
L=-\frac{d}{d x} x^{2} \frac{d}{d x}+2 x
$$

изучается аналогично оператору (4.7). Например, оператор $\widetilde{L}=F^{-1} L F$ действует по формуле (3.6), где $\tilde{q}(t)=2 e^{t}$. Решение уравнения $(2.7)$, где $\mu=$ $k^{2}+1 / 4$, принадлежащее $L^{2}$ на бесконечности, выражается опять через функцию Макдональда

$$
u_{k}(x)=x^{-1 / 2} K_{2 i k}(\sqrt{8 x}) .
$$

Согласно формулам (7.2.12) и (7.2.13) из [2], имеем

$$
u_{k}(x)=m(k) x^{-1 / 2+i k}+\overline{m(k)} x^{-1 / 2-i k}+O\left(x^{1 / 2}\right), \quad x \rightarrow 0,
$$

где

$$
m(k)=i \pi 2^{-1+i k}(\Gamma(1+2 i k) \operatorname{sh} 2 \pi k)^{-1} .
$$

Вычисляя $|m(k)|$ и используя (4.8), мы получаем, что теперь формула (1.4) принимает вид

$$
(U f)(k)=2 \pi^{-1} \sqrt{k \operatorname{sh} 2 \pi k} \int_{0}^{\infty} x^{-1 / 2} K_{2 i k}(\sqrt{8 x}) f(x) d x .
$$

Оператор $L$ не имеет собственных значений, так как функции $x^{-1 / 2} K_{2 p}(\sqrt{8 x})$ при $p \geqslant 0$ не принадлежат $L^{2}$ в окрестности точки $x=0$. Таким образом, как и в разд. 4.2, мы видим, что оператор $U$, определенный формулой (4.17), унитарен в пространстве $L^{2}\left(\mathbb{R}_{+}\right)$, а оператор $L$ имеет простой абсолютно непрерывный спектр $[1 / 4, \infty)$.

Из теоремы 2.1 вытекает, что

$$
\int_{0}^{\infty} a(x+y) y^{-1 / 2} K_{2 i k}(\sqrt{8 y}) d y=\lambda(k) x^{-1 / 2} K_{2 i k}(\sqrt{8 x})+\check{\lambda}(k) x^{-1 / 2} H_{2 i k}^{(2)}(i \sqrt{8 x})
$$

(функция Ганкеля $H_{2 i k}^{(2)}(i z)$ экспоненциально растет при $z \rightarrow \infty$ ) для некоторых постоянных $\lambda(k)$ и $\check{\lambda}(k)$. Так как интеграл в (4.18) (экспоненциально) убывает при $x \rightarrow \infty$, то обязательно $\check{\lambda}(k)=0$. Сравнивая асимптотики левой и правой частей в (4.18) при $x \rightarrow 0$ и используя соотношение $a(x) \sim x^{-1}$ при $x \rightarrow 0$, получаем, что число $\lambda(k)$ опять дается формулой (3.14). Таким образом, мы приходим к результату, аналогичному полученному в предыдущем разделе:

Предложение 4.2. Оператор Ганкеля $A$ с ядром (1.8) имеет простой абсолютно непрерывный спектр, совпадающий с интервалом $[0, \pi]$. Его нормированная собственная функция, отвечающая спектральной точке $\lambda=\pi(\operatorname{ch} \pi k)^{-1}$, дается формулой

$$
\psi_{k}(x)=2 \pi^{-1} \sqrt{k \operatorname{sh} 2 \pi k} x^{-1 / 2} K_{2 i k}(\sqrt{8 x}), \quad k>0 .
$$

Как побочный результат наших рассмотрений мы получили уравнение

$$
x^{1 / 2} \int_{0}^{\infty}(x+y)^{-1 / 2} K_{1}(\sqrt{x+y}) y^{-1 / 2} K_{2 i k}(\sqrt{y}) d y=\pi(\operatorname{ch} \pi k)^{-1} K_{2 i k}(\sqrt{x}) .
$$


Мы не нашли этого уравнения в литературе по специальным функциям. Отметим, однако, что оно может быть формально выведено из уравнения Шанкера (1.9), если использовать соотношение (формула (6.9.19) из [2])

$$
\lim _{\beta \rightarrow \infty} \Gamma(\beta+1) W_{-\beta, m}(x / \beta)=2 x^{1 / 2} K_{2 m}\left(2 x^{1 / 2}\right) .
$$

4.5. Оператор Карлемана $A$ очевидным образом попадает в изложенную выше схему. Теперь оператор $A$ коммутирует с оператором (1.7), где $\alpha=\beta=$ $\gamma=0$. Этот оператор имеет абсолютно непрерывный спектр кратности 2 , совпадающий с $[1 / 4, \infty]$. Его собственные функции $x^{-1 / 2+i k}$ для всех $k \in \mathbb{R}$ являются также собственными функциями оператора $A$. Соотношение между спектральными параметрами $\lambda$ и $k$ опять дается формулой (3.14), так что оператор $A$ имеет абсолютно непрерывный спектр кратности 2 , совпадающий с $[0, \pi]$.

\section{§5. Регулярные ядра}

5.1. Здесь мы рассмотрим ядра $a(x)$, которые быстро убывают при $x \rightarrow \infty$ и имеют конечные пределы при $x \rightarrow 0$. Положим $\gamma=2$ и будем различать случаи $\alpha>0, \beta$ произвольно и $\alpha=0, \beta>0$. Пусть $\alpha=1 / 4$ и $\beta=2$ соответственно в первом и втором случаях. Если $\alpha=1 / 4$, то решение уравнения (2.6) дается (см. разд. 4.1) формулой $b(r)=W_{-\beta, 1 / 2}(r)$, где $W_{-\beta, 1 / 2}-$ функция Уиттекера. Если $\alpha=0$ и $\beta=2$, то решение уравнения $(2.6)$ равно $b(r)=r^{1 / 2} K_{1}(\sqrt{8 r})$, где $K_{1}$ - функция Макдональда (см. разд. 4.4). Соответствующие функции $(2.5)$ экспоненциально убывают на бесконечности и имеют конечные пределы при $x \rightarrow 0$. Отсюда следует, что операторы $A$ компактны.

Пусть функция $\omega(x)$ определяется формулой $(3.5), \eta=\omega^{-1}$ и

$$
\tilde{q}(t)=-4^{-1}\left(\eta^{2}(t)+2 \eta(t)\right)^{-1}+\alpha \eta^{2}(t)+\beta \eta(t) .
$$

Так как $\eta(t) \sim e^{t} / 2$, то $\tilde{q}(t) \rightarrow+\infty$ при $t \rightarrow \infty$. Отсюда вытекает, что теперь операторы $\widetilde{L}$ и, следовательно, $L$ имеют дискретные спектры. Подчеркнем, что эти операторы по-прежнему определяются формулами (3.6) и (1.7) на функциях, удовлетворяющих соответственно граничным условиям (3.7) и (3.3).

Теорема 2.1 гарантирует, что операторы $A$ и $L$ имеют общие собственные функции. Вряд ли собственные функции оператора $L$ выражаются через стандартные специальные функции. Однако в их терминах мы можем вычислить собственные значения $\lambda_{1}, \lambda_{2}, \ldots$ оператора $A$. Действительно, пусть $\psi_{\mu} \in \mathscr{D}(L)$ и $L \psi_{\mu}=\mu \psi_{\mu}$. Тогда функция $\tilde{\psi}_{\mu}(t)=\left(\mathrm{F}^{-1} \psi_{\mu}\right)(t)$ удовлетворяет уравнению

$$
-\tilde{\psi}_{\mu}^{\prime \prime}(t)+\tilde{q}(t) \tilde{\psi}_{\mu}(t)=(\mu-1 / 4) \tilde{\psi}_{\mu}(t),
$$

где $\tilde{q}(t)$ - функция (5.1). При подходящей нормировке асимптотика $\tilde{\psi}_{\mu}(t)$ при $t \rightarrow \infty$ дается (см., например, книгу [7]) квазиклассической формулой

$$
\tilde{\psi}_{\mu}(t) \sim \tilde{q}(t)^{-1 / 4} \exp \left(-\int_{0}^{t} \tilde{q}(s)^{1 / 2} d s\right) .
$$

Отсюда следует, что $\tilde{\psi}_{\mu}(t) \sim e^{-t / 2} \exp \left(-4^{-1} e^{t}-\beta t\right)$ в первом случае и $\tilde{\psi}_{\mu}(t) \sim$ $e^{-t / 4} \exp \left(-2 e^{t / 2}\right)$ во втором. Возвращаясь к собственным функциям $\psi_{\mu}(x)$, находим, что

$$
\psi_{\mu}(x) \sim x^{-1-\beta} e^{-x / 2} \quad \text { и } \quad \psi_{\mu}(x) \sim x^{-3 / 4} e^{-\sqrt{8 x}}, \quad x \rightarrow \infty,
$$


соответственно в первом и втором случаях.

С другой стороны, используя асимптотики (4.2) и (4.16) для функции (1.8), мы видим, что

$$
\left(A \psi_{\mu}\right)(x) \sim x^{-1-\beta} e^{-x / 2} \int_{0}^{\infty} e^{-y / 2} \psi_{\mu}(y) d y
$$

И

$$
\left(A \psi_{\mu}\right)(x) \sim \sqrt{2 \pi} x^{-3 / 4} e^{-\sqrt{8 x}} \int_{0}^{\infty} \psi_{\mu}(y) d y
$$

при $x \rightarrow \infty$ соответственно в первом и втором случаях. Сравнивая эти соотношения с соотношениями $(5.2)$ и используя уравнение $A \psi_{\mu}=\lambda_{\mu} \psi_{\mu}$, получаем выражения для собственных значений оператора $A$ :

$$
\lambda_{\mu}=\int_{0}^{\infty} e^{-y / 2} \psi_{\mu}(y) d y \quad \text { и } \quad \lambda_{\mu}=\sqrt{2 \pi} \int_{0}^{\infty} \psi_{\mu}(y) d y
$$

соответственно в первом и втором случаях.

Таким образом, мы получили следующие результаты.

Предложение 5.1. Оператор Ганкеля $A$ с ядром

$$
a(x)=(x+2)^{-1} W_{-\beta, 1 / 2}(x+2)
$$

и дифберенциальный оператор (1.7) для $\gamma=2$ и $\alpha=1 / 4$ имеют общие собственные функции. Если $\psi_{\mu} \in \mathscr{D}(L), L \psi_{\mu}=\mu \psi_{\mu} u \psi_{\mu}(x)$ имеет первую из асимптотик (5.2) при $x \rightarrow \infty$, то $A \psi_{\mu}=\lambda_{\mu} \psi_{\mu}$, где $\lambda_{\mu}$ определяется первой из формул (5.3).

Предложение 5.2. Oператор Ганкеля $A$ с ядром

$$
a(x)=(x+2)^{-1 / 2} K_{1}(\sqrt{8(x+2)})
$$

и дифберенциальный оператор (1.7) для $\gamma=2, \alpha=0$ u $\beta=2$ имеют общие собственные функиии. Если $\psi_{\mu} \in \mathscr{D}(L), L \psi_{\mu}=\mu \psi_{\mu} u \psi_{\mu}(x)$ имеет вторую из асимптотик (5.2) при $x \rightarrow \infty$, то $A \psi_{\mu}=\lambda_{\mu} \psi_{\mu}$, где $\lambda_{\mu}$ определяется второй из бормул (5.3).

5.2. Наконец, мы рассмотрим ядро (1.5) для исключительных значений $\beta=$ $-l$, где $l=1,2, \ldots$ Более точно, мы сейчас положим

$$
a(x)=(-1)^{l-1}((l-1) !)^{-1} x^{-1} W_{l, 1 / 2}(x)=e^{-x / 2} L_{l-1}^{1}(x), \quad l=1,2, \ldots
$$

(здесь мы учли формулу (4.6)). Оператор Ганкеля $A$ с таким ядром имеет ранг $l$. Здесь мы покажем, как этот простой пример укладывается в описанную выше схему.

Спектральный анализ соответствующего оператора (4.7) остается таким же, как в разд. 4.2. Помимо абсолютно непрерывного спектра $[1 / 4, \infty)$, оператор $L$ имеет собственные значения $\mu_{n}=1 / 4-(l+1 / 2-n)^{2}$, где $n=1, \ldots, l$.

Однако вместо абсолютно непрерывного спектра оператор $A$ имеет нулевое собственное значение бесконечной кратности. Действительно, как и в разд. 4.3, теорема 2.1 дает уравнение $(4.12)$, где опять $\check{\lambda}(k)=0$. Заметим, что $a(x)$ и, следовательно, ввиду (4.3) интеграл в левой части формулы (4.12) имеют конечные пределы при $x \rightarrow 0$. Поэтому из $(4.3)$ следует, что обязательно $\lambda(k)=0$ для всех $k>0$. Таким образом, ядро оператора $A$ порождается функциями $x^{-1} W_{l, i k}(x), k>0$. 
Собственные функции $\psi_{n}(x)$, отвечающие ненулевым собственным значениям $\lambda_{n}$ оператора $A$, определяются формулой $(4.11)$, где $p=l+1 / 2-n$, $n<l+1 / 2$, а $\lambda_{n}$ находятся из уравнения (4.13):

$$
\int_{0}^{\infty} L_{l-1}^{1}(x+y) e^{-y} y^{p-1 / 2} L_{n-1}^{2 p}(y) d y=\lambda_{n} x^{p-1 / 2} L_{n-1}^{2 p}(x) .
$$

Вспомним, что $L_{p}^{\alpha}(x)$ - полином степени $p$ с коэффициентом $(-1)^{p} / p !$ при $x^{p}$. Сравнивая коэффициенты при наибольшей степени $x^{l-1}$ в левой и правой частях формулы (5.5) и учитывая формулу (4.14), получаем, что

$$
\lambda_{n}=(-1)^{n-l} \frac{(n-1) !}{(l-1) !} \int_{0}^{\infty} e^{-y} y^{p-1 / 2} L_{n-1}^{2 p}(y) d y=(-1)^{n-l} .
$$

Таким образом, мы пришли к следующему результату.

Предложение 5.3. Оператор Ганкеля $A$ с ядром (5.4) имеет ранг l. Его ненулевые собственные значения даются бормулой $\lambda_{n}=(-1)^{n-l}$, где $n=$ $1, \ldots, l$, а соответствующие собственные функиии $\psi_{n}(x)$ определяются $p$ венством (4.11), где $p=l+1 / 2-n$.

\section{ЛитерАТУРА}

[1] Н. И. Ахиезер, И. М. Глазман, Теория линейных операторов в гилъбертовом пространстве, Наука, М., 1966.

[2] Г. Бейтман, А. Эрдейи, Высшие трансцендентные функции. m. 1, 2, Наука, М., 1973, 1974.

[3] Н. Я. Виленкин, Специальные функции и теория представлений групп, Наука, M., 1965.

[4] T. Carleman, Sur les équations intégrales singulières à noyau réel et symetrique, Almqvist and Wiksell, 1923.

[5] W. Magnus, On the spectrum of Hilbert's matrix, Amer. J. Math., 72 (1950), 699-704.

[6] F. G. Mehler, Über eine mit den Kugel- und Cylinderfunctionen verwandte Function und ihre Anwendung in der Theorie der Elektricitätsvertheilung, Math. Ann., 18:2 (1881), 161-194.

[7] F. W. J. Olver, Asymptotics and Special Functions, Academic Press, 1974.

[8] В. В. Пеллер, Операторь Ганкеля и их приложения, НИЦ РХД, М.-Ижевск, 2005.

[9] M. Rosenblum, On the Hilbert matrix, I, II, Proc. Amer. Math. Soc., 9 (1958), 137-140, $581-585$.

[10] Э. Ч. Титчмарш, Разложения по собственным функииям, связанные с дифференциальными уравнениями второго порядка, m. 1, ИЛ, М., 1961.

[11] J. S. Howland, Spectral theory of operators of Hankel type. I, II, Indiana Univ. Math. J., 41:2 (1992), 409-426, 427-434.

[12] H. Shanker, An integral equation for Whittaker's confluent hypergeometric function, Proc. Cambridge Philos. Soc., 45 (1949), 482-483.

[13] D. R. Yafaev, Mathematical Scattering Theory. Analytic Theory, Amer. Math. Soc., Providence, RI, 2010.

IRMAR, Université de Rennes I Campus de Beaulieu e-mail: yafaev@univ-rennes1.fr

Поступило в редакцию 17 мая 2010 г. 